\title{
Raman Spectroscopy and Innovative Solar Cells - Optical Characterization of Molecules
}

\author{
Nikolaos F. Voudoukis
}

\begin{abstract}
Raman Effect is a phenomenon which results from the interaction of light and matter. Raman spectroscopy is the technique which applies this phenomenon to investigate the small world of microcrystals, microorganisms, microelectronics etc. Raman scattering is a non- invasive experimental method that detects the vibration of a molecule and identifies the characteristic spectral pattern (fingerprint) of it. Samples can be examined in as solids, liquids or vapors, in hot or cold states, in bulk, as microscopic particles, or as surface layers. It is described an experiment in which the purpose is to heat the powder of lead phthalocyanine $(\mathrm{PbPc})$ molecules (sample) in glass container so as to have PbPc free molecules in vapor phase. The study of PbPc in vapor phase is important as PbPc is deposited after being vaporized in a kind of innovative solar cells.
\end{abstract}

Index Terms - Raman Effect, Raman scattering, Raman spectroscopy, optical characterization, phthalocyanine PbPc, solar cells.

\section{INTRODUCTION}

Raman scattering is a non- invasive experimental method that detects the vibration of a molecule and identifies the characteristic spectral pattern (fingerprint) of it. Samples can be examined in as solids, liquids or vapors, in hot or cold states, in bulk, as microscopic particles, or as surface layers.

Raman Effect is a phenomenon which results from the interaction of light and matter. Raman spectroscopy is the technique which applies this phenomenon to investigate the small world of microcrystals, microorganisms, microelectronics etc. The phenomenon of inelastic scattering of light was first predicted theoretically by Smekal in 1923 and first observed experimentally (in liquids) by Raman and Krishnan in 1928, therefore it is referred as Raman Effect since then [1].

In the original experiment sunlight was focused by a telescope onto a sample which was either a purified liquid or a dust free vapor. A second lens was placed by the sample to collect the scattered radiation. A system of optical filters was used to show the existence of scattered radiation with an altered frequency of the incident light- the basic characteristic of Raman spectroscopy.

The scattering of light, as a result of its interaction with matter, can be classified as elastic (Rayleigh or Mie-Tyndall scattering) or inelastic (Raman or Brillouin scattering). In elastic scattering the scattered light is observed at the same frequency as the incident light. In the second case of inelastically scattered light, this is detected at different frequencies of the incident light and constitutes the Raman or Brillouin spectrum of the sample.

\section{THE RAMAN EFFECT}

Raman Effect results from the interaction of vibrational and / or rotational motions of molecules with the electromagnetic radiation, while Brillouin scattering involves the translational motion of molecules in liquids and solid and produce very small frequency shifts which has not yield yet important applications.

In Raman Effect inelastic scattering process the two photons are not identical and there is a net change in the state of the molecule. When the created photon is less energetic than the annihilated one, the scattered light is observed at a lower frequency than the incident light. This is Stokes Raman scattering. When the created photon is more energetic of the two, the Raman frequency will be higher than that of the laser and the anti-Stokes spectrum will be produced.

The scattering process described above is illustrated in Figure 1. The laser excitation at a frequency reappears as the relative strong Rayleigh line. The much weaker Raman "sidebands" are the result of inelastic scattering by a molecular vibration. The efficiencies of these scattering processes are very low. In order of magnitude the intensity of the Rayleigh line is about with respect to the incident excitation, while Raman features are at least another factor weaker copyright form and the form should accompany your final submission.

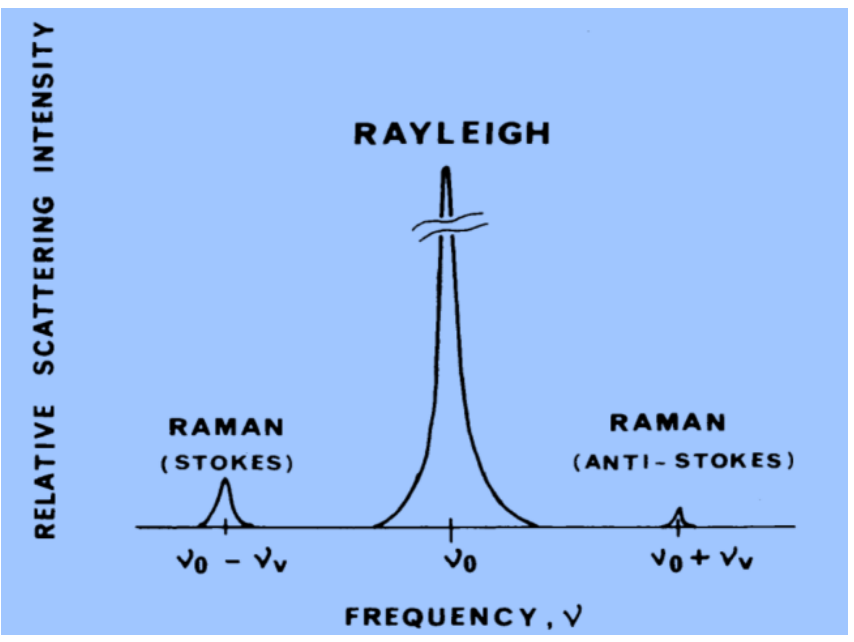

Fig. 1. Raman and Rayleigh scattering of excitation at a frequency $\mathrm{v}_{0}$. A molecular vibration in the sample is of frequency $\mathrm{v}_{\mathrm{v}}$

Raman scattering and infrared absorption are two of the main spectroscopic techniques to detect vibrations in molecules. They are used to provide information on chemical structures and physical forms, to identify substances from the characteristic spectral patterns 
(fingerprinting), and to determine quantitatively or semiquantitatively the amount of a substance in a sample.

The important characteristics of Raman microscopy are related to two fundamental optical considerations: the focusing of the incident laser excitation on the sample and the collection of the scattered light. Raman spectroscopy uses a single frequency of radiation to irradiate the sample and the radiation scattered from the molecule, which is different one vibrational unit of energy from the incident beam is detected.

\section{RAMAN SPECTROSCOPY- THEORY}

The theory of Raman spectroscopy will be further analyzed. To begin with, we will state the difference between absorption and scattering of the light. In Raman scattering we are interested only for the scattering of the light. The interaction of light and matter can lead to the absorption or scattering of light. The phenomenon of absorption of light by matter happens when the energy of the incident photon corresponds to the energy gap between the ground state of a molecule and the excited state.

The scattering of light happens when a light wave passes over a molecule and interacts and distorts the cloud of electrons around the nuclei. This phenomenon differs from the absorption's phenomenon as it does not promote any electron to an excited state of the static molecule. (Another difference is that the lifetime of the excited state is very short compared to most absorption processes. The entire process is completed within picoseconds or less.) More specifically, the light while passes over the molecule polarizes the electrons in a higher energy state. At that instant, the energy present in the light wave is transferred into the molecule.

This interaction can be considered as the formation of a very short-lived 'complex' between the light energy and the electrons in the molecule in which the nuclei do not have time to move appreciably. This results in a high energy form of the molecule with different electron geometry but without any large nuclear movement. This "complex" between the light and the molecule is not stable and the light is released immediately as scattered radiation. It is often called the virtual state of the molecule as we can see in the following figure.

Further the actual shape of the distorted electron arrangement will depend on how much energy is transferred to the molecule and hence is dependent on the frequency of the laser used. Thus, the laser defines the energy of the virtual state and the extent of the distortion. This virtual state is a real state of the transitory "complex" formed. The radiation is scattered as a sphere and not lost by energy transfer within the molecule or emitted at a lower energy. In addition there is a link between the polarization of the exciting and scattered photons which can be of value in assigning particular vibrations.

Raman and Rayleigh scattering are illustrated in the figure below. Rayleigh scattering is an elastic process (no change in the energy) and happens when the electron cloud relaxes without nuclear movement, therefore the light returns to the same energy state. Rayleigh scattering is the most usual scattering of photons therefore it is so intense. Raman scattering on the other hand is a much rarer event which involves only one in - of the photons scattered. This occurs when the light and the electrons interact and the nuclei begin to move at the same time. Since the nuclei are much heavier than the electrons, there is an appreciable change in energy of the molecule to either lower or higher energy depending on whether the process starts with a molecule in the ground state (Stokes scattering) or from a molecule in an excited vibrational state (anti-Stokes scattering).

More specifically, Stokes scattering happens when the molecule that is at the ground vibrational state absorbs energy and is being promoted to an excited vibrational state. Anti-stokes scattering happens when the molecule is at an excited vibrational state (probably due to thermal energy) and with the interaction to a photon, the molecule transfers part of its energy to that photon and rests at the ground state. At room temperature $\left(27^{\circ} \mathrm{C}\right.$ or $\left.300 \mathrm{~K}\right)$ the majority of the molecules are in low energy states - ground vibrational states, therefore the phenomenon of Anti-Stokes scattering is rare.

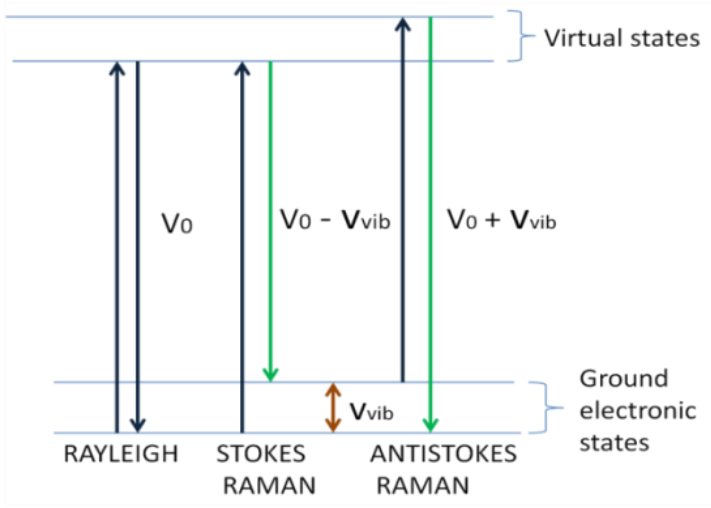

Fig. 2. Raman and Rayleigh scattering

In addition in Raman scattering, high energy radiation is used to excite the molecules and the difference in energy between the ground and the excited vibrational state of the molecule is measured by subtracting the energy of the scattered photon with that of the incident beam [2][3].

Raman spectroscopy deals with the analysis of the inelastically scattered light from atoms and molecules. Due to the interaction of light and matter, absorption or emission of photons are possible leading to changes of the frequency of the incident light.

The practical applications of Raman spectroscopy beyond pure science are

- $\quad$ Raman gas analyzers are used in medicine for realtime monitoring of anesthetic and respiratory gas mixtures during surgery.

- $\quad$ Spatially offset Raman spectroscopy (SORS), which is less sensitive to surface layers than conventional Raman, can be used to discover counterfeit drugs without opening their packaging, and for non-invasive monitoring of biological tissue.

- Raman spectroscopy can be used to investigate the chemical composition of historical documents with a noninvasive way.

This technique is applied mainly in physics and chemistry, apart from medicine and archeology mentioned 
before, as it can identify vibrational and rotational modes of a molecule by analyzing the Raman spectrum. This is what we want to find in our experiment the 'fingerprint' (the rotational and vibrational modes) of gas lead phthalocyanine $(\mathrm{PbPc})$ - free molecules.

$\mathrm{PbPc}$ contains 8 nitrogen atoms, 32 carbon atoms, 16 hydrogen atoms and one metal atom and its fundamental vibrational modes for Raman and infrared spectra are:

Гvib $(\mathrm{PbPc})=22 \mathrm{~A} 1(\mathrm{IR}, \mathrm{Ra})+41 \mathrm{E}(\mathrm{IR}, \mathrm{Ra})+21 \mathrm{~B} 1(\mathrm{Ra})$ $+21 \mathrm{~B} 2(\mathrm{Ra})+19 \mathrm{~A} 2, \mathrm{~A}$ is the symmetrical breathing mode and $\mathrm{B}, \mathrm{E}$ are the anti-symmetrical modes.

\section{DESCRIPTION OF AN EXPERIMENT - OPTICAL CHARACTERIZATION OF PBPC FREE MOLECULES}

It is described an experiment in which the purpose is to heat the powder of lead phthalocyanine $(\mathrm{PbPc})$ molecules (sample) in glass container so as to have $\mathrm{PbPc}$ free molecules in vapor phase. The study of $\mathrm{PbPc}$ in vapor phase is important as PbPc in a kind of innovative solar cells is deposited after being vaporized [4]. Therefore the information of structural characterization of $\mathrm{PbPc}$ in vapor phase that is given by Raman spectrum is considerable as it provides with information before the electrical characterization of the solar cells ( $\mathrm{PbPc}$ on GaAs substrates). The spectral pattern of $\mathrm{PbPc}$ in vapor phase is taken and the analysis of the vibration modes of $\mathrm{PbPc}$ free molecules is examined.

The experimental set- up is consisted by a laser Argon cations able to produce a strong beam, lenses and reflecting mirrors to focus and enhance the beam on the sample by aligning the beam to the focus point, a Dilor Charge-Couple Device (CCD) which is a very sensitive detector of light, cooled to minimize the background noise so that we have a clearer vibrational spectrum. The sample is tied around with a ribbon of glass fibers that inside has a wire of wolfram. The wire is afterwards connected with the power supply and provided with current so as to raise its temperature. The reason that the the sample is heated, is that we want the $\mathrm{PbPc}$ powder to evaporate so that the $\mathrm{PbPc}$ molecules are in gas phase and therefore free.

Other main device is the vacuum pump that is connected with the sample so that a vacuum of approximately $8 * 10^{-8}$ mbar is achieved and no air is in the sample when we take the spectrum of the molecules. In addition thermocouple is used to measure the temperature of the sample. The sample of the powder $\mathrm{PbPc}$ is important to reach the sublimation temperature of $400^{\circ} \mathrm{C}$ to $500^{\circ} \mathrm{C}$ so that we have free molecules.

Contamination of the equipment may lead to false results therefore we have to be very careful with the lenses and the glass containers by using plastic gloves. Also protective glasses must be used for eyes protection from the laser radiation during the experiment. Another factor that needs time and trials is the alignment of the Raman equipment in the sample and a calibration.

The set-up of the experiment contains:

1. Argon ${ }^{+}$laser producing green line of 514,5 $\mathrm{nm}$. (Generally, Argon ion lasers emit at 13 wavelengths through the visible, ultraviolet, and near-visible spectrum, including: $351.1 \mathrm{~nm}, 363.8 \mathrm{~nm}, 454.6 \mathrm{~nm}, 457.9 \mathrm{~nm}, 465.8 \mathrm{~nm}, 476.5$ $\mathrm{nm}, 488.0 \mathrm{~nm}, 496.5 \mathrm{~nm}, 501.7 \mathrm{~nm}, 514.5 \mathrm{~nm}, 528.7 \mathrm{~nm}$, $1092.3 \mathrm{~nm}$ with the most prominent and most used wavelengths in the argon laser are the $514.5 \mathrm{~nm}$ green line which - used in this experiment- and the $488.0 \mathrm{~nm}$ blue line.)

2. Lens- mirror of the alignment of the laser beam to the sample as well as to reflect the beam back to the focus point so as to increase the sensitivity which is low as the sample is in the gas phase.

3. Charge - Coupled Device, detector of the light, cooled with liquid nitrogen. Raman scattering has a very low signal therefore the background noise of the instrumentation has to be minimized therefore is used liquid nitrogen to eliminate the noise of CCD.

Glass containers can be used for the sample. Glass as water is a strong absorber of infrared radiation and they are both week scatterers in Raman spectroscopy which makes them (and especially the glass container used) suitable for the technique. The purpose is to heat the PbPc powder so it sublimates to gas phase so as to have free molecules and at that moment take the spectrum - the fingerprint of the free molecules PbPc.

For the sample can be used:

a. Glass cell (maybe two glass cells for comparison because the optical quality of glass is good we do not have so much scattered light and therefore background).

b. Metallic rings to have high temperature around the holes so as to avoid sublimation of the molecules on the holes. If with the use of metallic rings it is not achieved to eliminate the phenomenon of sublimation on the holes, heat gun is a second solution.

c. Ribbon of glass fibers tied in grid so that the sample is heated homogeneously with inside the ribbon wire of wolfram. The wire of wolfram is twiddled (two wires) so that it has less resistance and raise the temperature of the sample far more.

d. Power supply of current-voltage to raise the temperature of the sample to approximately $570^{\circ} \mathrm{C}$ and no more as the quartz glass has a softening temperature above $580^{\circ} \mathrm{C}$.

e. Thermocouple of nickel chrome and nickel aluminum.

It is very important that the sample has homogeneity so that any focal point produces the same results. This is the reason to try to heat homogeneously the sample using the glass ribbon with the wolfram wire inside, the metallic rings and the heat gun.

As it is investigated the gas phase of $\mathrm{PbPc}$ it is important to know that the number of molecules in gas phase per volume of container is considerable, therefore it is advisable to use very long glass container.

Interesting information is that, as we are trying to take the spectrum of $\mathrm{PbPc}$ free molecules, we may observe sometimes cosmic rays in the spectrum. These appear abnormally strong with a narrow bandwidth in weak spectrum. Other false lines in a spectrum can be produced by room lights (so during the experiment the lights have to be closed). 
It is very important that the glass container is clean from fingerprints which may cause fluorescing. The sample must be tied with the glass ribbon in grid very carefully so that there are no large holes and the cell is well covered in order to be heated homogenously. Current have to be applied through the wolfram wire inside the glass ribbon with a small step so that we don't have huge and rapid differences in temperature with the danger of breaking the glass of the sample. When the temperature reach $565^{\circ} \mathrm{C}$ we did not raise more the temperature as the molecules of lead phthalocyanine would be destroyed- burnt.

The main problem at this phase of the procedure is the sublimation of the molecules on the open holes. That is difficult to avoid as the difference in temperatures in those small holes permit the molecules to stack on them so the beam could not pass through. One solution for this problem is to use heat gun for extra heat on those holes.

The Stokes scattering is produced by the vibration of the molecules (phonons) that have absorbed light (photons) of the laser with energy $\mathrm{E}=\mathrm{h} * \mathrm{c} / \lambda, \lambda=514,5 \mathrm{~nm}=\mathrm{E}=2,4 \mathrm{eV}$. After this absorption the molecules change the vibration mode in a more energetic one so we have a shift in their frequency. The scattered light - photons which now has less energy and therefore frequency is collected by the CCD (Charge Coupled Device). The difference in the intensity of the Raman Scattered light in the peaks is analogous to a difference in the intensity of the vibration of the molecules. The scattering is measured as light detected by the spectrometer and the maximum amount of light detected is the highest point on the trace. Raman scattering is presented only as the Stokes spectrum (and not as an anti- Stokes spectrum) and is given as a shift in energy from the energy of the laser beam.

Every vibration of the molecule has a specific Raman frequency therefore from the peaks we have in our spectrum we can identify the vibration mode that the molecules have. The spectrum do not start to measure from $(0,0)$ as in this point we have the Rayleigh line which is the elastic scattered light of the molecules and is very intense so the CCD will be damaged. Therefore, the most used spectrums recorded shifts down to $200 \mathrm{~cm}^{-1}$ and no lower (but the modern equipment of the technique can record shifts down $50 \mathrm{~cm}^{-1}$ ) as this range includes the most modes which are characteristic of the molecule and the Raman equipment is protected.

The cell is in vacuum so the spectrum counts only the Raman shift of lead phthalocyanine molecules in gas phase, and not contaminators (molecules of air etc).

The intensities of the bands in the Raman spectrum are dependent on the nature of the vibration being studied and on instrumentation and sampling factors. Modern instruments are be calibrated to remove the instrument factors- noise.)

In order to characterize the Raman spectrum of vapor PbPc DFT (Density Functional Theory) calculations have to be done so as to predict the band positions of the groups of it. An important parameter for the use of DFT calculations is that these predict spectra of single molecules in vapor phase as our PbPc molecule. Recorded spectra of DFT calculations of solids and liquids can have band shifts due to molecular interactions.

\section{REsUlts}

The graph of $\mathrm{PbPc}$ in vapor phase is taken by Raman experiment. The excitation laser line is 514,5 - green line with power $5,4 \mathrm{~W}$. The graph is edited after fitting the Raman peaks in vapor and then again edited by origin for precised determination of the peeks. The peaks were fitted taking in consideration to DFT calculations and therefore the peaks were determined by the vibrational modes. The first number of every peek is the one observed in our experiment and the second the vibrational mode assumed by fitting the peaks in consideration to the DFT calculations.

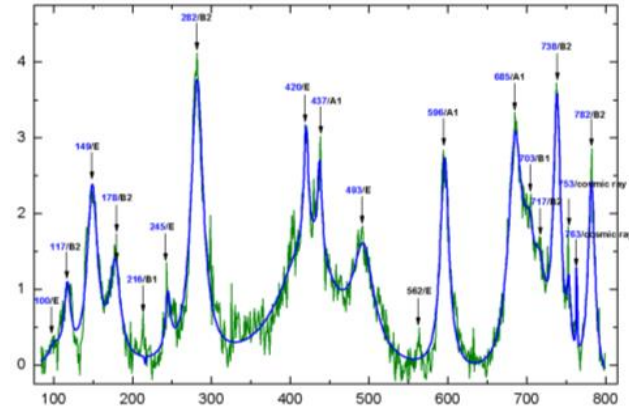

Fig. 3. Raman spectrum of $\mathrm{PbPc}$ in vapor phase: Intensity (a.u) vs Raman shift $\left(\mathrm{cm}^{-1}\right)$

In this graph the position given is the position of the Raman peak with an estimated divergence, the amplitude of the peak and the width of the peak. Our spectrum has a range of modes from $100 \mathrm{~cm}^{-1}$ to $800 \mathrm{~cm}^{-1}$. We can see 17 Raman peeks therefore 17 vibrations of the molecule. Peaks of 753 and 763 wavelength may be probably cosmic rays due to their narrow width. Another explanation of narrow Raman peaks is that $\mathrm{PbPc}$ is in vapor phase with low pressure but these explanations would lead to generally narrow peaks a fact that does not apply to the peaks of the graph therefore cosmic rays observed in our spectrum is the most possible fact. The criteria of the fitting are

- the mode frequency of the peaks,

- the mode intensity and

- $\quad$ the mode symmetry.

There are differences in the intensities of the experimental peaks and those expected by the DFT calculations. An explanation of this is given by the fact the PbPc is a large molecule with a rich Raman spectrum contrary to Si that has simply Raman spectrum with a single peak in $521 \mathrm{~cm}^{-1}$ therefore it is possible to observe differences in the frequencies and intensities of the spectrum if there are small discrepancies in the composition of the material.

After fitting the Raman peeks it is observed that there is identification on many vibrational modes of the DFT calculations to the spectrum and that a considerable number of Raman peeks have been closely identified in $\mathrm{PbPc}$ powder of the experimental data.

For further explanation we can see in the following figure: $\mathrm{N} 1$ is the nitrogen atom joined to the central metal and to 
this bond is referred the $\mathrm{Pb}-\mathrm{N} 1$ and $\mathrm{C}-\mathrm{N} 2$ the nitrogen atom not joined to the central metal. In addition, we can understand and see which are referred as $\mathrm{C}-\mathrm{C}, \mathrm{C}-\mathrm{N}, \mathrm{Pb}-\mathrm{N}$ (the connected atoms in the $\mathrm{PbPc}$ molecule). The bond and the atoms are also clearly depicted in the $\mathrm{PbPc}$ figure (following figure). Generally the symbols $\alpha, \beta, \gamma, \delta$ are given to the atoms to describe that they begin as is oindole units from the N1. In Pcs we have only $\alpha$ and $\beta$ connections.

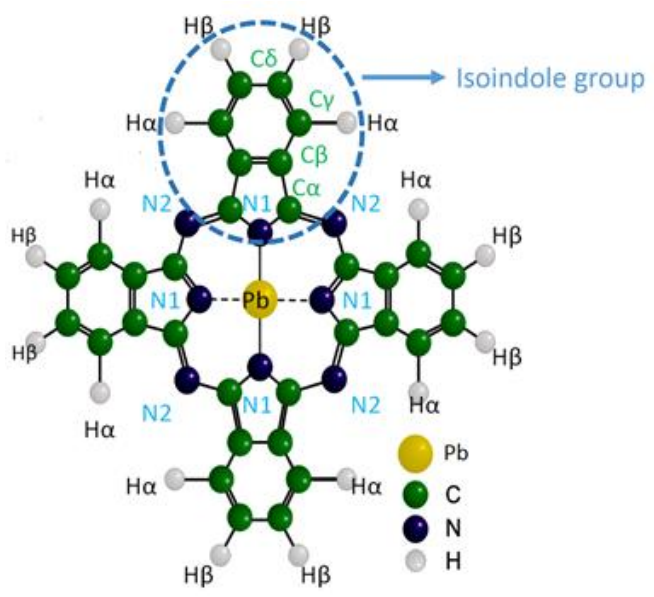

Fig.4. PbPc molecule and its bonds

The figure of the $\mathrm{PbPc}$ molecule clearly depicts the internal bonds of the atoms of the molecule and their positions. The $\mathrm{PbPc}$ molecule is non planar as (lead) ion is larger than the central cavity therefore lies above the molecule of phthalocyanine. This is shown in the second image of Figure 5.

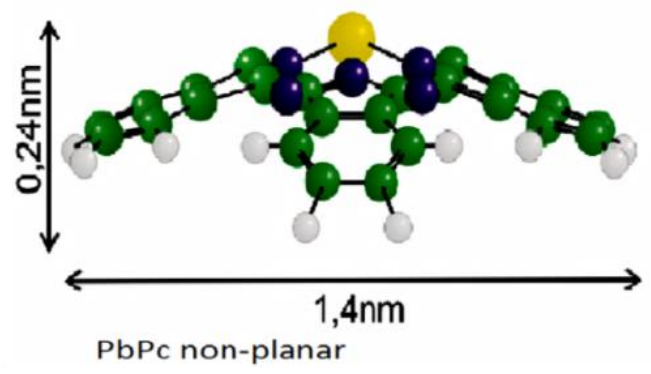

Fig.5. PbPc non- planar

The symmetry notation is from the group theory [5]. The $\mathrm{PbPc}$ molecule corresponds to the 4-fold rotational $\mathrm{z}$-axis $\mathrm{C}_{4}$ of which we have the symmetry notation $A_{1}, A_{2}, B_{1}, B_{2}$ and E. A is the symmetrical breathing mode and B, E the antisymmetrical modes corresponding to special bonds its time.

TABLE I: CHARACTER TABLE OF $\mathrm{C}_{4 \mathrm{~V}}$ POINT GROUP (PbPc)

\begin{tabular}{|c|c|c|c|c|c|c|}
\hline & $\mathrm{C}_{4 \mathrm{v}}$ & $\mathrm{E}$ & $\mathrm{C}_{2}$ & $2 \mathrm{C}_{4}$ & $2 \sigma_{\mathrm{v}}$ & $2 \sigma_{\mathrm{d}}$ \\
\hline \multirow{5}{*}{ 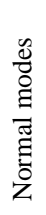 } & $\mathrm{A}_{1}$ & 1 & 1 & 1 & 1 & 1 \\
\hline & $\mathrm{A}_{2}$ & 1 & 1 & 1 & -1 & -1 \\
\hline & $\mathrm{B}_{1}$ & 1 & 1 & -1 & 1 & -1 \\
\hline & $\mathrm{B}_{2}$ & 1 & -1 & -1 & 1 & 1 \\
\hline & $\mathrm{E}$ & 2 & -2 & 0 & 0 & 0 \\
\hline
\end{tabular}

Where $\mathrm{E}$ is the intensity consists of no charge, $\mathrm{C}_{2}$ is horizontal rotational axis passing from $\sigma_{v}$, where $\sigma_{v}$ is the vertical axis along the $x-y$ direction of the molecule and $\sigma \mathrm{d}$ is the diagonal axis lying between the $\sigma v$ planes. $\mathrm{Cn}$ is the rotation around axis passing through the central atom by $360 / \mathrm{n}$ degrees; therefore $\mathrm{C}_{4}$ is the rotation by $360 / 4$ degrees $=90$ degrees. The figure below shows the rotational axis described.

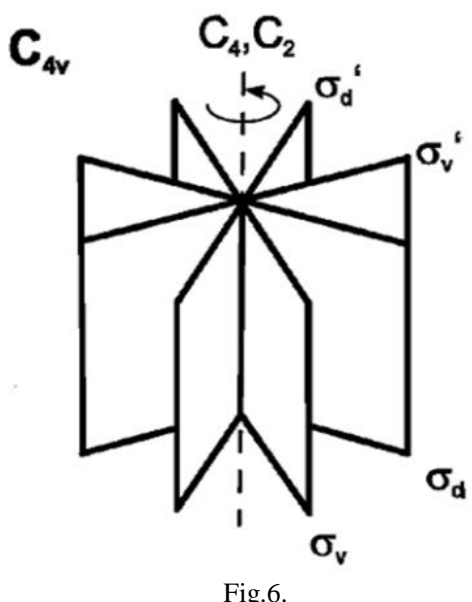

The vibrational modes A,B,E are the Mulliken symbols and their meaning is :

- A, B Non-degenerate (single) mode: one set of atom displacements. Symmetric (A) or antisymmetric (B) to inversion (-1).

- E Doubly degenerate mode. Two sets of atom displacements, symmetric or anti-symmetric.

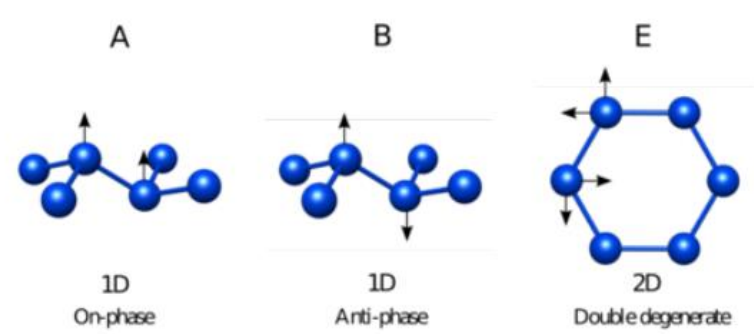

Fig.7. A,B,E vibrational modes

\section{CONCLUSION}

The analysis of the spectrum of free- molecule $\mathrm{PbPc}$ gives many identical assignments to both DFT calculations as well as their vibrational modes, but further experimental research should be done (so as the molecules of PbPc are free -larger container- no intermolecular interactions among the molecules and with no condensation on the windows of the container) and more research on the identification of the assignments with Density Functional Theory.

Simple explanations are given on what the vibrational modes of the PbPc molecule are. Raman spectroscopy it is used for identifying the vibrational modes of free molecule $\mathrm{PbPc}-$ vapor phase (from its Raman spectrum) in comparison with the theoretical calculations of Density Functional Theory (DFT). According to those DFT calculations it is done a new assignment which is in agreement with the experimental results.

Fitting results of vibrational mode frequencies of $\mathrm{PbPc}$ in vapor phase proposed in comparison with the results of 
Density Functional Theory (DFT) and mode assignment Generally, the theoretical calculations of Density Functional Theory may give a depiction of a vibration close to the truth but it cannot be done for every molecule, furthermore only the experimental Raman spectrum can characterize the molecule with certainty [6]. That means that only the experimental method of Raman scattering can provide us with the certain vibration modes.

\section{REFERENCES}

[1] E. Smith, G. Dent, Modern Raman Spectroscopy, A practical approach, Wiley, United Kingdom, 2005

[2] M.-S. Liao, S. Scheiner, Electronic structure and bonding in metal phthalo-cyanines, Metal $=\mathrm{Fe}, \mathrm{Co}, \mathrm{Ni}, \mathrm{Cu}, \mathrm{Zn}, \mathrm{Mg}$, Chemistry and Biochemistry Faculty Publications, 2001

[3] Lawrence L. Kazmerski, "Photovoltaics characterization: A survey of diagnostic measurements", Journal of Materials Research, 1998

[4] N. Voudoukis, "Photovoltaic Technology and Innovative Solar Cells" EJECE, European Journal of Electrical and Computer Engineering, Vol. 2, No. 1, January 2018

[5] Y. Zhang and X. Zhang: "Comparative density functional theory study of the structures and properties of metallophthalocyanines of group IV B", Vibrational Spectroscopy, vol.40, (2006), pg. 289-298

[6] W.E. Smith, G. Dent, Modern Raman Spectroscopy: A practical approach, Wiley publications Wiley publications, 2005

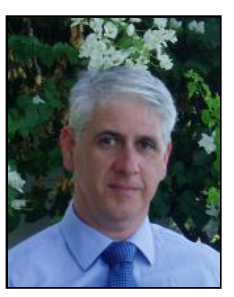

Nikolaos Voudoukis received a BSc degree in Physics from Athens National University, Greece, in 1991, a BSc in Electrical and Computer Engineering from the National Technical University of Athens, Greece, in 2012, his MSc degree in Electronics and Telecommunications from Athens National University, in 1993, and his $\mathrm{PhD}$ degree from Athens National University, in 2013. He has worked as telecommunication engineer in Greece, as teacher and Assistant Director at a high school and as a parttime Lecturer at the School of Pedagogical \& Technological. Education, Athens, Greece. He is IEEE member. Dr. Voudoukis now is with National Technical University of Athens, Greece. 